\title{
Instant sedative effect of acupuncture at GV20 on the frequency of electroencephalogram $\alpha$ and $\beta$ waves in a model of sleep deprivation
}

\author{
JIA LI $^{1}$, XIAO RAN ${ }^{1}$, CHAO CUI $^{1}$, CHAO XIANG $^{1}$, AO ZHANG $^{1,2}$ and FENG SHEN ${ }^{1}$ \\ ${ }^{1}$ College of Acupuncture and Orthopaedics, Hubei University of Chinese Medicine/Hubei Provincial Collaborative Innovation \\ Center of Preventive Treatment by Acupuncture and Moxibustion, Wuhan, Hubei 430061; ${ }^{2}$ College of Acupuncture, \\ Moxibustion and Tuina of Gansu University of Chinese Medicine, Lanzhou, Gansu 730000, P.R. China
}

Received August 3, 2016; Accepted November 22, 2017

DOI: $10.3892 /$ etm.2018.6123

\begin{abstract}
Sleep deprivation (SD) adversely affects brain function and is accompanied by frequency-dependent changes in electroencephalograms (EEGs). Recent studies have suggested that acupuncture is an emerging alternative therapy for SD. However, the involvement of the frequency of EEG $\alpha$ and $\beta$ waves in the protective effect of acupuncture against SD remains unknown. The present study investigated the instant effect of acupuncture at GV20 on insomnia by analyzing the frequency of $\alpha$ and $\beta$ waves using electroencephalography in a model of sleep deprivation. A total of 16 rats (Wistar; male; weight, $340 \pm 10 \mathrm{~g}$ ) were divided randomly into four groups (4 rats per group) to create a rat model of sleep deprivation using the modified multiple platform method in the GV20 group, the sham acupoint group and the model group. After $72 \mathrm{~h}$ of sleep deprivation for these three groups and normal feeding for the blank group, the EEG data of all four groups were documented. Following the initial measurement, the GV20 group was treated by acupuncture at GV20 and the sham acupoint group was treated at the sham acupoint, and their EEGs were recorded during the treatment. The frequency of $\alpha$ and $\beta$ waves of all EEG data were analyzed. Prior to intervention, the GV20 group, the sham acupoint group and the model group exhibited no significant differences in $\alpha$ and $\beta$ wave frequencies; however, the $\alpha$ wave frequency of
\end{abstract}

Correspondence to: Dr Ao Zhang, College of Acupuncture, Moxibustion and Tuina of Gansu University of Chinese Medicine, 35 Dingxi Road, Lanzhou, Gansu 730000, P.R. China

E-mail: 5495537764@qq.com

Dr Feng Shen, College of Acupuncture and Orthopaedics, Hubei University of Chinese Medicine/Hubei Provincial Collaborative Innovation Center of Preventive Treatment by Acupuncture and Moxibustion, 1 Tanhualin Road, Wuhan, Hubei 430061, P.R. China E-mail: 40052958@qq.com

Key words: GV20, acupuncture, insomnia, electroencephalogram, sedative effect these three groups was significantly decreased compared with the blank group $(\mathrm{P}<0.05)$, whereas the $\beta$ wave frequency of these three groups was significantly increased compared with the blank group $(\mathrm{P}<0.05)$. This suggested that sleep deprivation affected the frequency of brain waves and enhanced the excitability of the cerebral cortex. During acupuncture treatment with retained needle conditioning, the GV20 group indicated a significant increase in $\alpha$ wave frequency $(\mathrm{P}<0.05)$, as well as a significant decrease in $\beta$ wave frequency compared with prior to treatment $(\mathrm{P}<0.05)$, whereas the sham acupoint group exhibited no significant changes. The present findings from a rat model of sleep deprivation suggested that acupuncture treatment at GV20 may reduce the excitability of the brain cortex. Due to its sedative effect, treatment at GV20 may be considered for the treatment of insomnia and related symptoms.

\section{Introduction}

The primary symptoms of insomnia include difficulty in falling asleep, being easily awoken and unable to fall asleep again and being kept awake throughout the night (1). In Traditional Chinese Medicine, this disorder is called 'Bu Mei' (2). Insomnia may negatively impact the quality of the individuals everyday life $(3,4)$. The prevalence of insomnia disorder is $\sim 10-20 \%$ in the USA (5), with $\sim 50 \%$ of cases having a chronic cause. Insomnia may result in a diminished cognitive ability, including attention deficit, poor memory and impaired decision making (4). Insomnia symptoms are characterized by prolonged sleep latency and difficulty staying asleep as long as desired (4). Benzodiazepines are commonly prescribed for short-term relief of anxiety and insomnia symptoms, however certain patients continue taking these drugs and become long-term benzodiazepine users (6). Compared with drug therapy, acupuncture has been demonstrated to exhibit improved effects and cause fewer adverse reactions, however it may still lead to local allergies, local muscle tension and pain caused by needling or allergic reactions to the metal used $(7,8)$. The most common side effects associated with benzodiazepines are sedation, dizziness, weakness and unsteadiness (9). A previous randomized 
placebo-controlled study on the efficacy and safety of acupuncture [at Baihui (GV20), Yintang (EX-HN3), bilateral ear Shenmen, Sishencong (EX-HN1) and Anmian (EX)] for the short-term treatment of primary insomnia, revealed that electroacupuncture was superior to placebo acupuncture (10). A recent study has reported that the augmented acupuncture formula (points LI4, LIV3, EX-HN3, GV20, LU7 and KID6) was more effective for the treatment of depression and improved the sleep quality in patients who already had depression, compared with the standard acupuncture formula (points LI4, LIV3, EX-HN3 and GV20) (11). Based on this GV20 is frequently used for the acupuncture treatment of insomnia (12). Previous studies of acupuncture treatment have primarily focused on subjectively reported symptoms of insomnia. Consequently, they were not able to observe the mechanisms of acupuncture's effect on insomnia. In the present study, electroencephalogram (EEG) will be used to assess changes in the brain activity in real time. These measures will objectively examine real-time physiological changes due to acupuncture (13). To explore the mechanism of GV20 acupuncture treatment on insomnia, a rat model of sleep deprivation was constructed and the instant effects of acupuncture at GV20 on EEGs was analyzed by assessing the change of frequency of EEG $\alpha$ and $\beta$ waves.

\section{Materials and methods}

Experimental animals and grouping. A total of 16 Wistar male rats (age, 12 months; weight, 340 $\pm 10 \mathrm{~g}$ ) were obtained from the Experimental Animal Research Center of Hubei (Wuhan, China; no. SCXK(E)2008-0005). The rats were allowed free access to food and water and were housed under a controlled temperature $\left(23 \pm 2^{\circ} \mathrm{C}\right)$ and humidity (40-60\%), with $12 \mathrm{~h}$ of artificial light per day. Following adaptive feeding for 1 week prior to the experiment, all rats were divided into the blank group, the GV20 group, the sham acupoint group and the model group (4 rats per group). All experimental procedures complied with the guidelines of the Principles of Laboratory Animal Care (14) and the legislation of the People's Republic of China for the Use and Care of Laboratory Animals (15). The experimental protocols were approved by the Animal Experimentation Ethics Committee of the Hubei University of Traditional Chinese Medicine (Wuhan, China).

Experiment instruments. An MP150 multichannel physiological signal recorder (Biopac Systems Inc., Goleta, CA, USA) and cuboid-shaped wire mesh $(100 \times 90 \times 15 \mathrm{~cm})$ were used in the present study. A circular sink $(120 \times 80 \mathrm{~cm})$ was purchased from Chengdu Taimeng Technology Co., Ltd. (Chengdu, China) and an acupuncture needle $(0.20 \times 13 \mathrm{~mm})$ was obtained from Beijing Hanyi Medical Apparatus and Instruments Center (Beijing, China).

Modeling method. The modified multiple platform method (MMPM) using the platform technique (16) was applied in the present experiment, using 12 platforms (17). The cuboid-shaped wire mesh was placed into a circular sink, and the sink was filled with water until the water was $\sim 1 \mathrm{~cm}$ below the surface of the grid the rats were on. The 12 rats (GV20, sham acupoint and model groups) were settled on the cross points and were forced to grasp the wire to maintain a standing position to prevent falling. These rats could freely move around the sink on the mesh. When the rats fell asleep, they would immediately fall into the water due to the relaxation in their muscle tension. As a consequence of this, rats would need to swim to the wire mesh and stand on it again. The temperature of the room was maintained at $25 \pm 1^{\circ} \mathrm{C}$. During the total $72 \mathrm{~h}$ of producing the model, water in the sink was exchanged once every $12 \mathrm{~h}$. While exchanging water, the rats were placed in cages to be fed. The feeding time was limited to $1 \mathrm{~min}$ and the cages were kept shaking to prevent the rats from falling to sleep.

The blank group was reared in a normal environment (temperature, $25 \pm 1^{\circ} \mathrm{C}$ ) with a $12 \mathrm{~h} \mathrm{light/dark} \mathrm{cycle.} \mathrm{In} \mathrm{order}$ to eliminate the influence of swimming and the limitation of feeding time, the blank group was also fed once every $12 \mathrm{~h}$, with the feeding time limited to $1 \mathrm{~min}$. Rats were forced to swim for $10 \mathrm{sec}$ after every feed.

Intervention methods. Following $72 \mathrm{~h}$ of modeling, EEGs of all 16 rats were measured and recorded immediately (120 sec for each rat). Subsequently, the acupuncture treatment and synchronized measuring of EEGs was initiated instantly (within $120 \mathrm{sec}$ of treating and recording for each rat).

In order to obtain EEG measurements, sterilized needle electrodes were inserted into the subcutaneous tissues of rat's heads in an awakened state. The positive electrode was $0.3 \mathrm{~cm}$ to the right of the vertex of parietal bone and the negative electrode was $0.3 \mathrm{~cm}$ to the left of the vertex of parietal bone. The reference electrode was placed on the subcutaneous tissue of the neck. During the measurement and the recording, all the rats were captured and fastened in the same way with homemade cloths.

For acupuncture treatment, the acupoints were based on the 'Map of the Experimental Animal Acupuncture Points,' which was formulated by the Experimental Acupuncture Institute of China Association of Acupuncture and Moxibustion (18). For the GV20 group, the needle was horizontally inserted at GV20 at the vertex of the rat's parietal bone (19) via an even reinforcing-reducing method, as described previously (20). The needle was rotated bidirectionally within $90^{\circ}$ at a speed of $2 \mathrm{~Hz}$. The needle was retained for $120 \mathrm{sec}$, and rotated once every $40 \mathrm{sec}$. For the sham acupoint group, the needle was inserted at the sham acupoint $(0.15 \mathrm{~cm}$ to the right of the vertex of the rat's parietal bone), and the manipulation was the same as that of the GV20 group.

Statistical analysis. Data were analyzed with the SPSS software, version 20.0 (IBM Corp., Armonk, NY, USA) and expressed as the mean \pm standard deviation. One-way analysis of variance (ANOVA) followed by Tukey's post hoc test was performed for analysis. $\mathrm{P}<0.05$ was considered to indicate a statistically significant difference.

\section{Results}

Behavior observation. During the first $24 \mathrm{~h}$ of modeling, the GV20 group, the sham acupoint group and the model group were acting normally and rats crept on the wire and reacted strongly when being captured. Between 25-48 h, the majority 


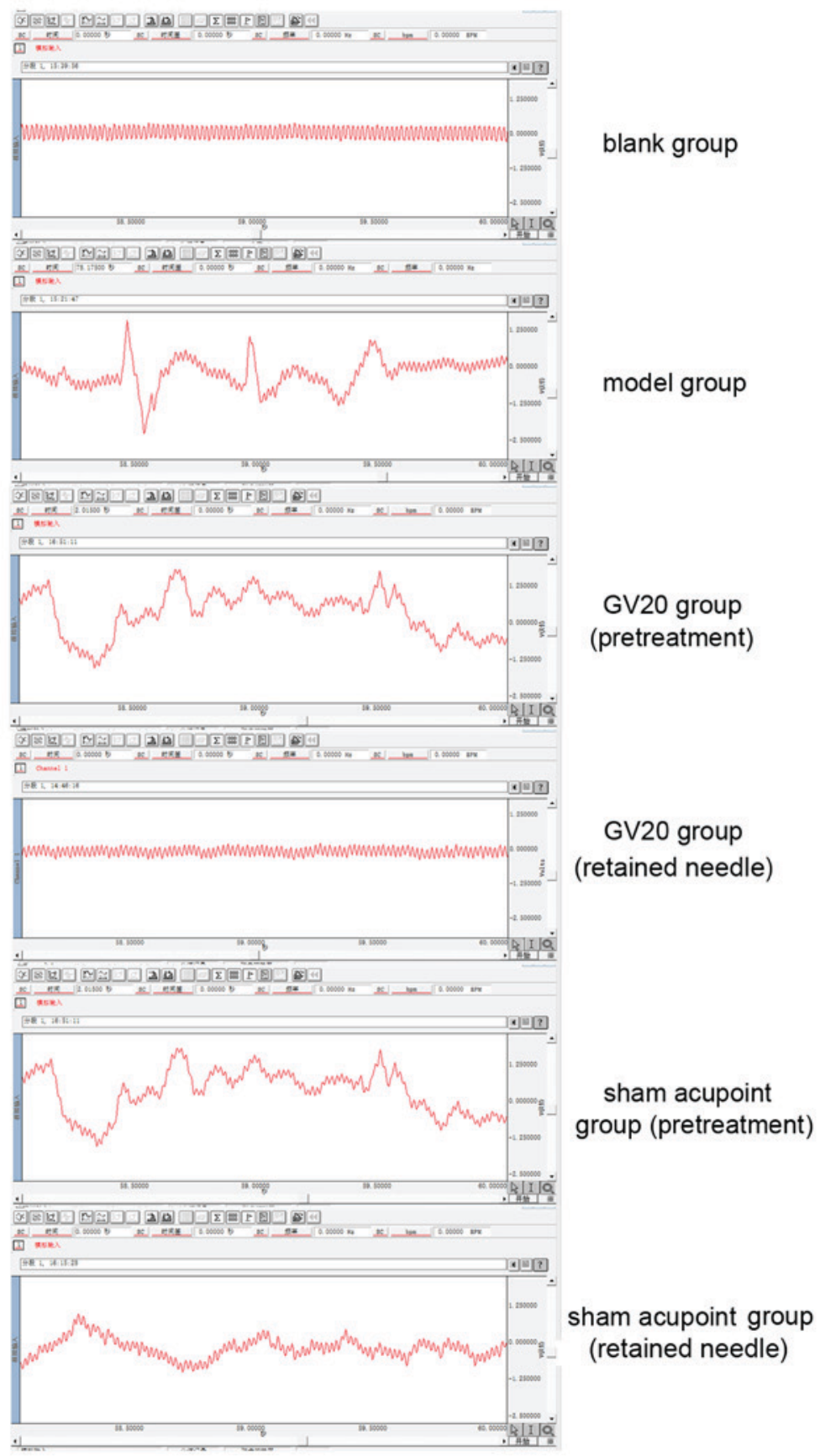

Figure 1. Electroencephalograms from rats in the blank, model, GV20 (pretreatment and retained needle) and Sham acupoint (pretreatment and retained needle) groups.

of the rats demonstrated tiredness: Rats crouched and shook instead of creeping and occasionally fell into the water and had to swim back to the wire. Furthermore, rats began to show signs of a reduced appetite when being fed, as they preferred sleeping to eating, their cages had to be kept shaking to prevent them from sleeping. Between 49-72 h, all three groups of rats were observed and appeared much thinner compared with prior to the modeling experiment, their fur was wet and messy, and their ears, eyes and the extremities of limbs had turned pink or pale. In addition, the majority of the rats demonstrated signs of excitement and mania. Rats shook a lot, tended to fight each other, fiercely struggled when being captured for feeding and had good appetites when being offered food and water. During the 72-h modeling period of the three groups, the rats in the blank group, which were reared under standard conditions, acted normally.

Within $10 \mathrm{sec}$ of acupuncture treatment, rats in the GV20 group fell into sleep instantly, breathing stably and were crouched with their eyes closed. The sham acupoint group remained awake within $10 \mathrm{sec}$ of the sham acupoint acupuncture treatment and were still shaking, aggressively; their characteristics were similar to the model group at the same time.

Analysis of EEG. EEGs from all rats and the analysis of the frequency of $\alpha$ and $\beta$ waves are demonstrated in Fig. 1 and Tables I and II. As indicated in Fig. 1, compared with the blank group, the model, GV20 (pretreatment) and 
Table I. Frequency of $\alpha$ and $\beta$ waves of the blank, model, GV20 (pretreatment) and sham acupoint (pretreatment) groups.

Wave frequency, $\mathrm{mHz}$

\begin{tabular}{lccc}
\cline { 3 - 3 } Group & $\mathrm{n}$ & $\alpha$ & $\beta$ \\
\hline GV20 (pretreatment) & 4 & $10.1208 \pm 0.4955^{\mathrm{a}}$ & $32.3789 \pm 0.7171^{\mathrm{a}}$ \\
Sham acupoint (pretreatment) & 4 & $10.5254 \pm 0.4064^{\mathrm{a}}$ & $32.6520 \pm 2.1659^{\mathrm{a}}$ \\
Model & 4 & $10.0009 \pm 0.0857^{\mathrm{a}}$ & $33.0258 \pm 0.7713^{\mathrm{a}}$ \\
Blank & 4 & $12.2918 \pm 0.1842$ & $21.5471 \pm 0.7273$
\end{tabular}

${ }^{a} \mathrm{P}<0.05$ vs. the corresponding blank group. Data are presented as mean \pm standard deviation.

Table II. Frequency of $\alpha$ and $\beta$ waves in the GV20 and sham acupoint groups.

\begin{tabular}{clcc}
\hline & & \multicolumn{2}{l}{ Wave frequency, mHz } \\
\cline { 4 - 4 } Group & measurement taken & $\alpha$ & $\beta$ \\
\hline GV20 & & & \\
Rat 1 & Pretreatment & 10.1936 & 32.6975 \\
& Retained needle & $12.2616^{\mathrm{a}}$ & $25.4447^{\mathrm{a}}$ \\
Rat 2 & Pretreatment & 10.5781 & 32.6994 \\
& Retained needle & $12.2057^{\mathrm{a}}$ & $23.0262^{\mathrm{a}}$ \\
Rat 3 & Pretreatment & 10.2926 & 32.9251 \\
& Retained needle & $12.3499^{\mathrm{a}}$ & $22.8959^{\mathrm{a}}$ \\
Rat 4 & Pretreatment & 9.4188 & 31.1205 \\
& Retained needle & $12.2616^{\mathrm{a}}$ & $22.9197^{\mathrm{a}}$ \\
Sham acupoint & & & \\
Rat 1 & Pretreatment & 10.5558 & 31.9311 \\
& Retained needle & $10.7676^{\mathrm{b}}$ & $31.5172^{\mathrm{b}}$ \\
Rat 2 & Pretreatment & 10.8959 & 29.7769 \\
& Retained needle & $10.4773^{\mathrm{b}}$ & $29.3446^{\mathrm{b}}$ \\
Rat 3 & Pretreatment & 10.6971 & 35.6291 \\
& Retained needle & $10.2877^{\mathrm{b}}$ & $36.3446^{\mathrm{b}}$ \\
Rat 4 & Pretreatment & 9.9528 & 33.2436 \\
& Retained needle & $9.6955^{\mathrm{b}}$ & $33.4247^{\mathrm{b}}$ \\
\hline
\end{tabular}

${ }^{\mathrm{a}} \mathrm{P}<0.05$ vs. the corresponding pretreatment; ${ }^{\text {b}} \mathrm{P}<0.05$ vs. the corresponding retained needle in the GV20 group.

sham acupoint (pretreatment) groups demonstrated similar characteristics in their EEG data. The EEGs indicated relatively disordered shapes, irregular waves, low amplitude and unclear amplitude modulations, which were similar to EEGs of panasthenia (21). Paroxysmal spines, sharp waves, spine-slow synthetical waves and sharp-slow synthesis waves were observed on the EEGs. The frequency of $\alpha$ and $\beta$ waves in all four groups were compared using one-way ANOVA followed by Tukey's post hoc test. Significant differences among the four groups were observed ( $\alpha$ wave frequency, $\mathrm{F}=39.919$ and $\mathrm{P}<0.05 ; \beta$ wave frequency, $\mathrm{F}=61.170$ and $\mathrm{P}<0.05$; Table I). The frequency of $\alpha$ waves of the GV20 (pretreatment), sham acupoint (pretreatment) and the model groups were significantly reduced compared with the blank group $(\mathrm{P}<0.05)$. Conversely, the frequency of $\beta$ waves of the GV20 (pretreatment), sham acupoint (pretreatment) and model groups were significantly increased compared with the blank group $(\mathrm{P}<0.05)$. These findings indicated that the production of the sleep deprivation model was successful. Comparison of the frequency of $\alpha$ and $\beta$ waves in the GV20 and sham acupoint groups (pretreatment and retained needle) using one-way ANOVA followed by Tukey's post hoc test indicated the differences among the two groups were statistically significant ( $\alpha$ wave frequency, $\mathrm{F}=32.225$ and $\mathrm{P}<0.05 ; \beta$ wave frequency, $\mathrm{F}=53.352, \mathrm{P}<0.05$; Table II), allowing a comparative study for acupuncture treatment to be performed.

For the GV20 group, the differences in frequency of $\alpha$ and $\beta$ waves at pretreatment compared with frequencies when the needle was retained at GV20 were statistically significant $\left(\mathrm{P}_{\alpha}=0.003\right.$ and $\left.\mathrm{P}_{\beta}=0.001\right)$, and when the needle was retained, the EEG frequency of $\alpha$ waves was significantly increased whereas the frequency of $\beta$ waves was significantly decreased. In the sham acupoint group, the differences in the frequency of $\alpha$ and $\beta$ waves between pretreatment and when the needled was retained were not statistically significant $\left(\mathrm{P}_{\alpha}=0.237\right.$ and $\left.\mathrm{P}_{\beta}=0.966\right)$. These results indicated that the acupuncture treatment at GV20 was able to relieve the central excitement of insomnia in rats, improve the frequency of $\alpha$ waves and decrease the frequency of $\beta$ waves, providing an instant sedative effect. Conversely, sham acupuncture treatment demonstrated no notable curative effect.

\section{Discussion}

GV20 is located on the vertex of the body and is also called the 'intersection point of Three Yang and Five Channels' because GV20 is the intersection point of Governor vessel, foot-taiyang meridian, foot-shaoyang meridian, hand-shaoyang meridian and foot-jueyin meridian (22). Various studies support the sedative effects of GV20 in treating insomnia and relieving symptoms $(23,24)$. A previous study has indicated that treatment using GV20 regulates the body state in patients with insomnia by repairing neurons, regulating the content of 5-hydroxyidnoleacetic acid, improving the blood supplement to the brain and enhancing the activity of acetylcholinesterase in the brain (25). 
As an objective index in evaluating brain function, EEG is an intuitive, sensitive, specific and easy method for analysis, which makes it a popular method in research and for the diagnosis of mental disorders and central nervous system diseases (26). In addition, EEG is generally applied in the study of psychology, scientific research and the detection of anesthesia (27). In literature regarding sleep disorders, EEGs are typically used. For example, polysomnography is an important detection method with EEG being its most important index (28). Currently, EEG is used in comparing changes in patients with insomnia before and after their treatment to determine the clinical effects (29). In cases where the normal conscious body is in a quiet and relaxed state, predominantly $\alpha$ waves of $8-13 \mathrm{~Hz}$ are indicated in EEGs (30). However, in cases where the body is in an anxious, nervous or manic state, $\beta$ waves of $14-30 \mathrm{~Hz}$ are primarily observed (31). In conscious patients with insomnia, the EEG data may indicate the phenomena of slowing $\alpha$ waves, lower amplitude and a lack of amplitude modulation, in addition to increased $\beta$ wave rhyme (32-36). Therefore, the increase in the proportion of $\alpha$ waves is a valuable index in evaluating the change of insomnia symptoms, and changes in $\beta$ waves may be used in determining anxiety and mania in patients with insomnia.

In the present study, when sleep deprivation was achieved, the frequency of $\alpha$ waves in the GV20 (pretreatment), sham acupoint (pretreatment) and model groups were decreased compared with the blank group. However, the frequency of $\beta$ waves in the GV20 (pretreatment), sham acupoint (pretreatment) and model groups were increased compared with the blank group. Results demonstrated that after $72 \mathrm{~h}$ of sleep deprivation, rats with insomnia were anxious and excited. When the needle was retained at GV20, the frequency of $\alpha$ waves were improved and the frequency of $\beta$ waves was decreased, which suggested this treatment provided an instant sedative effect.

In conclusion, the present findings indicated that acupuncture treatment at GV20 may be an effective application in treating insomnia symptoms, such as anxiety and mania. The present study may be meaningful for designing clinical acupuncture treatment plans for patients with insomnia.

\section{Acknowledgements}

The present study was supported by Hubei Province Scientific and Technological Research Projects (grant nos. 20162006 and 2016CFB221).

\section{Competing interests}

The authors declare that they have no competing interests.

\section{References}

1. Ohayon MM: Epidemiology of insomnia: What we know and what we still need to learn. Sleep Med Rev 6: 97-111, 2002.

2. Deng A, Jiang Re and Ma Z: Literature study on distribution features of tcm syndromes and syndrome elements of insomnia. Chin Med Mod Dis Education China 13: 147-149, 2015 (In Chinese).

3. Mishima K, DiBonaventura Md and Gross H: The burden of insomnia in Japan. Nat Sci Sleep 7: 1-11, 2015.
4. Buysse DJ: Insomnia. JAMA 309: 706-716, 2013

5. Burman D: Sleep disorders: Insomnia. FP Essent 460: 22-28, 2017.

6. Kaufmann CN, Spira AP, Depp CA and Mojtabai R: Long-term use of benzodiazepines and nonbenzodiazepine hypnotics, 1999-2014. Psychiatr Serv 69: 235-238, 2018.

7. Xie ZY and Wu Xi: Observation on the efficacy of acupoint massage plus moxibustion for refractory insomnia. J Acupuncture Tuina Sci 13: 44-48, 2015 (In Chinese).

8. Sok SR, Erlen JA and Kim KB: Effects of acupuncture therapy on insomnia. J Adv Nurs 44: 375-384, 2003.

9. Uzun S, Kozumplik O, Jakovljević M and Sedić B: Side effects of treatment with benzodiazepines. Psychiatr Danub 22: 90-3, 2010.

10. Yeung WF, Chung KF, Zhang SP, Yap TG and Law AC: Electroacupuncture for primary insomnia: A randomized controlled trial. Sleep 32: 1039-1047, 2009.

11. Wen X, Wu Q, Liu J, Xu Z, Fan L, Chen X, He Q, Ma R, Wu Y, Jiang S, et al: Randomized single-blind multicenter trial comparing the effects of standard and augmented acupuncture protocols on sleep quality and depressive symptoms in patients with depression. Psychol Health Med 23: 375-390, 2018.

12. Kalavapalli R and Singareddy R: Role of acupuncture in the treatment of insomnia: A comprehensive review. Complement Ther Clin Pract 13: 184-193, 2007.

13. Han KH, Kim SY and Chung SY: Effect of acupuncture on patients with insomnia: Atudy protocol for a randomized controlled trial. Trials 15: 403, 2014.

14. Bayne K: Revised guide for the care and use of laboratory animals available. american physiological society. Physiologist 39: 199 208-211, 1996.

15. Kong Q and Qin C: Analysis of current laboratory animal science policies and administration in China. ILAR J 51: e1-e11, 2009.

16. Cohen HB and Dement WC: Sleep: Changes in threshold to electroconvulsive shock in rats after deprivation of 'paradoxical' phase. Science 150: 1318-1319, 1995.

17. Machado RB, Hipólide DC, Benedito-Silva AA and Tufik S: Sleep deprivation induced by the modified multiple platform technique: Quantification of sleep loss and recovery. Brain Res 1004: 45-51, 2004.

18. Yin CS, Jeong HS, Park HJ, Baik Y, Yoon MH, Choi CB and Koh HG: A proposed transpositional acupoint system in a mouse and rat model. Res Vet Sci 84: 159-65, 2008.

19. Yin CS, Jeong HS, Park HJ, Baik Y, Yoon MH, Choi CB and Koh HG: A proposed transpositional acupoint system in a mouse and rat model. Res Vet Sci 84: 159-65, 2008.

20. Ding N, Jiang J, Lu M, Hu J, Xu Y, Liu X and Li Z: Manual acupuncture suppresses the expression of proinflammatory proteins associated with the nlrp3 inflammasome in the hippocampus of samp8 mice. Evid Based Complement Alternat Med 2017: 3435891, 2017.

21. Neznamov GG, Bochkarev VK, Siuniakov SA and Grishin SA: Characteristics of ladasten effect in neurasthenia patients with various eeg parameters. Eksp Klin Farmakol 71: 18-25, 2008 (In Russian).

22. Shen EY, Chen FJ, Chen YY and Lin MF: Locating the acupoint baihui (GV20) beneath the cerebral cortex with MRI reconstructed 3D neuroimages. Evid Based Complement Alternat Med 2011: 362494, 2011.

23. Montakab H and Langel G: The effect of acupuncture in the treatment of insomnia. Clinical study of subjective and objective evaluation. Schweiz Med Wochenschr Suppl 62: 49-54, 1994 (In French).

24. Spence DW, Kayumov L, Chen A, Lowe A, Jain U, Katzman MA, Shen J, Perelman B and Shapiro CM: Acupuncture increases nocturnal melatonin secretion and reduces insomnia and anxiety: A preliminary report. J Neuropsychiatry Clin Neurosci 16: 19-28, 2004.

25. Jiang YJ: Electro acupuncture Du20 and Du24 on Cognitive Impairment after Stoke (unpublished PhD thesis). Fujian University of Chinese Medicine, Fuzhou, 2011.

26. Fu K, Qu J, Chai Y and Zhou T: Hilbert marginal spectrum analysis for automatic seizure detection in EEG signals. Biomedical Signal Processing Control 18: 179-185, 2015.

27. Zhang C: Analysis of electrocephalogram and electrocephafulctuo technology on 'rigid-gentle syndrome differentiation' of psychosomatic diseases (unpublished $\mathrm{PhD}$ thesis). China Academy of Chinese Medicine Science, 2013.

28. Kempfner J, Sorensen GL, Sorensen HB and Jennum P: Automatic REM sleep detection associated with idiopathic rem sleep Behavior Disorder. Conf Proc IEEE Eng Med Biol Soc 2011: 6063-6: 2011 
29. Perlis ML, Kehr EL, Smith MT, Andrews PJ, Orff H and Giles DE: Temporal and stagewise distribution of high frequency EEG activity in patients with primary and secondary insomnia and in good sleeper controls. J Sleep Res 10: 93-104, 2001.

30. Coll MP, Press C, Hobson H, Catmur C and Bird G: Crossmodal classification of mu rhythm activity during action observation and execution suggests specificity to somatosensory features of actions. J Neurosci 37: 5936-5947, 2017.

31. Li N, Wang P, Deng B, Wei XL, Che YQ, Jia CH, Guo Y and Chao W: Influence of acupuncture of Zusanli (ST 36) on connectivity of brain functional network in healthy subjects. Zhen $\mathrm{Ci}$ Yan Jiu 36: 278-87, 2011 (In Chinese).

32. Perlis ML, Merica H, Smith MT and Giles DE: Beta EEG activity and insomnia. Sleep Med Rev 5: 363-374, 2001.

33. Perlis ML, Smith MT, Andrews PJ, Orff $\mathrm{H}$ and Giles DE: Beta/Gamma EEG activity in patients with primary and secondary insomnia and good sleeper controls. Sleep 24: 110-117, 2001.
34. Krystal AD, Edinger JD, Wohlgemuth WK and Marsh GR: NREM sleep EEG frequency spectral correlates of sleep complaints in primary insomnia subtypes. Sleep 25: 630-640, 2002.

35. Goljahani A, Bisiacchi P and Sparacino G: An EEGLAB plugin to analyze individual EEG alpha rhythms using the 'channel reactivity-based method'. Comput Methods Programs Biomed 113: 853-861, 2014.

36. Merica H, Blois R and Gaillard JM: Spectral characteristics of sleep EEG in chronic insomnia. Eur J Neurosci 10: 1826-1834, 1998. 